\title{
Wrinkling transition in quenched disordered membranes at two loops
}

\author{
O. Coquand ${ }^{1,2, *}$ and D. Mouhanna ${ }^{1, \dagger}$ \\ ${ }^{1}$ Sorbonne Université, CNRS, Laboratoire de Physique Théorique de la Matière Condensée, LPTMC, F-75005 Paris, France \\ ${ }^{2}$ Institut für Materialphysik im Weltraum, Deutsches Zentrum für Luft- und Raumfahrt, Linder Höhe, 51147 Köln, Germany
}

(Received 8 November 2020; accepted 5 February 2021; published 1 March 2021)

\begin{abstract}
We investigate the flat phase of quenched disordered polymerized membranes by means of a two-loop, weak-coupling computation performed near their upper critical dimension $D_{u c}=4$, generalizing the one-loop computation of Morse et al. [D. C. Morse et al., Phys. Rev. A 45, R2151 (1992); D. C. Morse and T. C. Lubensky, Phys. Rev. A 46, 1751 (1992)]. Our work confirms the existence of the finite-temperature, finite-disorder wrinkling transition, which has been recently identified by Coquand et al. [O. Coquand et al., Phys. Rev. E 97, 030102(R) (2018)] using a nonperturbative renormalization group approach. We also point out ambiguities in the two-loop computation that prevent the exact identification of the properties of the novel fixed point associated with the wrinkling transition, which very likely requires a three-loop order approach.
\end{abstract}

DOI: 10.1103/PhysRevE.103.L031001

Introduction. The critical and, more generally, the longdistance equilibrium statistical physics of pure, homogeneous systems is now widely understood. By contrast, quenched, random heterogeneities, such as defects or impurities, inevitably present in most real physical systems, are known to give rise to a wide spectrum of new phenomena. Quenched disordered membranes occupy a special place (see, e.g., [1]), as their most famous physical realizations seem to bring out the physical effects of both random bonds and random fields; see [2-8] for reviews. For instance, in a series of experiments beginning in the early 1990s, Mutz et al. [9] and then Chaieb et al. $[10,11]$ showed that upon cooling below the chain melting temperature, photoinduced partially polymerized vesicles made of diacetylenic phospholipid undergo a transition from a smooth structure, at high polymerization, to a wrinkled structure, at low polymerization, with randomly frozen normals that could characterize a glassy phase. This transition and the resulting wrinkled phase have been conjectured to result from the joint effects of random heterogeneities on both the internal metric and the curvature of the membrane [12] that bear formal similarities with, respectively, random bonds and random fields in magnetic systems; see below. More recently, in the context of the rapidly growing defect engineering [13-16] of graphene $[17,18]$, it has been shown that by thoroughly damaging a clean sheet of this material with a laser beam, it is possible to induce a crystal-to-glass transition giving rise to a vacancy-amorphized graphene structure [19-21]. Here, also, the inclusion of lattice defects-foreign adatoms or substitutional impurities - is expected to lead, in addition to metric alterations, to a rearrangement of $\mathrm{sp}^{2}$-hybridized carbon atoms into nonhexagonal structures and, thus, to the generation of nonvanishing curvature structures, showing again that the un-

\footnotetext{
*oliver.coquand@dlr.de

${ }^{\dagger}$ mouhanna@1ptmc.jussieu.fr
}

derlying physics could rely on the coexistence of the two kinds of disorder.

Disordered membranes also stand out from the crowd by the theoretical investigations to which they have been subjected. Stimulated by the work of Mutz et al. [9] on partially polymerized vesicles, the first attempt to probe the effects of disorder in membranes was realized by Nelson and Radzihovsky [12,22], who focused their study on the role of disorder in the preferred metric. Performing a weakcoupling expansion near the upper critical dimension $D_{u c}=4$, they showed that for $D<4$, while the disorder is irrelevant and the renormalization group (RG) flow is driven toward the disorder-free fixed point $P_{4}$ at any finite temperature, an instability close to $T=0$ could be accompanied by a diverging Edwards-Anderson correlation length, leading to a glassy phase. Then, Radzihovsky and Le Doussal [23], by employing a large embedding dimension $d$ expansion, confirmed such a possibility, finding an instability of the flat phase toward a spin-glass-like phase. However, no quantitative or qualitative empirical confirmation of this scenario has been provided. Morse et al. [24,25] then reconsidered the weak-coupling analysis of Refs. [12,22] within an approach that included both metric and curvature disorders. They confirmed the irrelevance of the disorder in $D<4$, but showed that the presence of curvature disorder gives rise to a new and vanishing temperature fixed point $P_{5}$ that is stable with respect to randomness but unstable with respect to the temperature. Somewhat disappointing from the point of view of the search for a new exotic phase, these works have been followed mainly by mean-field approximations involving either short-range [23,26-31] or long-range [32,33] disorder, leading to speculate about the existence of a glassy phase at any temperature and for large enough disorder; see [34] for a review. Again, no confirmation of this conjecture has been provided. However, very recently, an approach based on the nonperturbative renormalization group (NPRG), following those performed on disorder-free membranes 
[35-40], was realized by Coquand et al. [41] on the model considered by Morse and Lubensky displaying both metric and curvature disorders. Their main result has been to identify a novel finite-temperature, finite-disorder fixed point $P_{c}$ that was once unstable, and thus associated with a second-order phase transition and making the $T=0$ fixed point fully attractive provided $T<T_{c}$. This study has allowed the identification of three distinct universal scaling behaviors [42] corresponding both qualitatively and quantitatively to those observed in the experiments of Chaieb et al. [10,11].

Whereas the NPRG results have been challenged within a recent self-consistent screening approximation approach [34], they have been confirmed within a large- $d$ approach performed at next-to-leading order in $1 / d$ [43], although in a model involving only curvature disorder. In this controversial context, it was compelling to further investigate the model of Morse et al. [24,25]. In that respect, an essential feature of the novel fixed point $P_{c}$ found in [41] is that its coordinates near $D_{u c}=4$ differ only from those of the vanishing-temperature fixed point $P_{5}$ by terms of order $\epsilon^{2}$, with $\epsilon=4-D$, strongly suggesting that $P_{c}$ could also be identified within a perturbative $\epsilon$ expansion up to this order. This is the reason why, in this Letter, we investigate quenched disordered membranes at two loops in the vicinity of the upper critical dimension, extending both the one-loop computation of Morse et al. performed 30 years ago $[24,25]$ at the next order and the recent two-loop computation of Coquand et al. [44] (see also [45]) on disorder-free membranes to the disordered case. We derive the RG equations, analyze them, and provide the critical quantities, notably the anomalous dimension $\eta$, at order $\epsilon^{2}$. Our approach unambiguously confirms the existence of the once-unstable fixed point $P_{c}$, characterizing a phase transition between a high-temperature phase controlled by the disorderfree fixed point $P_{4}$ and a low-temperature phase controlled by the vanishing-temperature, finite-disorder fixed point $P_{5} .{ }^{1}$ It nevertheless also reveals a drawback of the perturbative approach at two-loop order, which manifests through the impossibility to exactly identify the location and properties of the fixed points $P_{5}$ and $P_{c}$ at this order; we argue that this should very likely be raised by a three-loop order computation.

The action. A membrane is modelized by a $D$-dimensional surface embedded in a $d$-dimensional Euclidean space. A point on the membrane is thus identified by $D$-dimensional vector $\mathbf{x}$ and a configuration of the membrane in the Euclidean space is described through the embedding $\mathbf{x} \rightarrow \mathbf{R}(\mathbf{x})$, with $\mathbf{R} \in \mathbb{R}^{d}$. In the flat phase, we define the average position of a point $\mathbf{x}$,

$$
\mathbf{R}^{0}(\mathbf{x})=[\langle\mathbf{R}(\mathbf{x})\rangle]=\zeta x_{i} \boldsymbol{e}_{i},
$$

where the $\boldsymbol{e}_{i}$ form an orthonormal set of $D$ vectors and $\zeta$ is the stretching factor taken to be one in what follows. In Eq. (1), $[\cdot]$ and $\langle\cdot\rangle$ denote averages over disorder and thermal fluctuations, respectively. The fluctuations around the configuration (1) are parametrized by writing $\mathbf{R}(x)=\mathbf{R}^{0}(\mathbf{x})+$

\footnotetext{
${ }^{1}$ Note that here we consider the flat phase of membranes; also, the high-temperature phase discussed here should not be confused with the crumpled phase of membranes.
}

$\mathbf{u}(\mathbf{x})+\mathbf{h}(\mathbf{x})$, with $\mathbf{h} \cdot \boldsymbol{e}_{i}=0$. The fields $\mathbf{u}$ and $\mathbf{h}$ represent $D$ longitudinal-phonon—and $d-D$ transverse-flexuralmodes, respectively. The long-distance, effective action is given by $[24,25]$

$$
\begin{aligned}
\mathcal{S}= & \int d^{D} x\left\{\frac{\kappa}{2}[\Delta \boldsymbol{h}(\mathbf{x})]^{2}+\frac{\lambda}{2} u_{i i}(\mathbf{x})^{2}+\mu u_{i j}(\mathbf{x})^{2}\right. \\
& \left.-\boldsymbol{c}(\mathbf{x}) \cdot \Delta \boldsymbol{h}(\mathbf{x})-\sigma_{i j}(\mathbf{x}) u_{i j}(\mathbf{x})\right\} .
\end{aligned}
$$

In Eq. (2), the first term represents the curvature energy with bending rigidity $\kappa$, while the second and third terms represent the elastic energies, with $u_{i j}$ being the strain tensor which, truncated to its most relevant part, reads $u_{i j} \simeq$ $\frac{1}{2}\left[\partial_{i} u_{j}+\partial_{i} u_{j}+\partial_{i} \boldsymbol{h} \cdot \partial_{j} \boldsymbol{h}\right] ; \lambda$ and $\mu$ are the Lamé coefficients. The fourth and fifth terms in Eq. (2) represent disorder fields $\boldsymbol{c}$ and $\sigma_{i j}$ that couple, respectively, to the curvature $\Delta \boldsymbol{h}$-thus linearly to $\boldsymbol{h}$ as a random field ${ }^{2}$-and to the strain tensor $u_{i j}$ - thus quadratically to $\boldsymbol{h}$ as a random mass. These fields are chosen to be short-range quenched Gaussian ones with zero-mean value and variances given by $[24,25]$

$$
\begin{aligned}
{\left[c_{i}(\mathbf{x}) c_{j}\left(\mathbf{x}^{\prime}\right)\right] } & =\Delta_{\kappa} \delta_{i j} \delta^{(D)}\left(\mathbf{x}-\mathbf{x}^{\prime}\right), \\
{\left[\sigma_{i j}(\mathbf{x}) \sigma_{k l}\left(\mathbf{x}^{\prime}\right)\right] } & =\left(\Delta_{\lambda} \delta_{i j} \delta_{k l}+2 \Delta_{\mu} I_{i j k l}\right) \delta^{(D)}\left(\mathbf{x}-\mathbf{x}^{\prime}\right),
\end{aligned}
$$

where $I_{i j k l}=\frac{1}{2}\left(\delta_{i k} \delta_{j l}+\delta_{i l} \delta_{j k}\right)$, with $i, j, k, l=1 \ldots D$. Stability considerations require that the coupling constants $\kappa, \mu$, and $\lambda+(2 / D) \mu$, as well as $\Delta_{\kappa}, \Delta_{\mu}$, and $\Delta_{\lambda}+(2 / D) \Delta_{\mu}$, are positive.

Disorder averages are performed through the replica trick, which leads to the effective action [24,25],

$$
\begin{aligned}
S= & \int d^{D} x\left\{\frac{\widetilde{Z}_{\alpha \beta}}{2} \Delta \boldsymbol{h}^{\alpha}(\mathbf{x}) \Delta \boldsymbol{h}^{\beta}(\mathbf{x})+\frac{\tilde{\lambda}_{\alpha \beta}}{2} u_{i i}^{\alpha}(\mathbf{x}) u_{j j}^{\beta}(\mathbf{x})\right. \\
& \left.+\widetilde{\mu}_{\alpha \beta} u_{i j}^{\alpha}(\mathbf{x}) u_{i j}^{\beta}(\mathbf{x})\right\},
\end{aligned}
$$

where Greek indices are associated with the $n$ replica. In Eq. (4), we have rescaled the fields $\boldsymbol{h} \mapsto T^{1 / 2} Z^{1 / 2} \kappa^{-1 / 2} \boldsymbol{h}$, $\boldsymbol{u} \mapsto T Z \kappa^{-1} \boldsymbol{u}$, where $Z$ is a field renormalization, and introduced the running coupling constants $\widetilde{\lambda}=\lambda T Z^{2} \kappa^{-2}$, $\tilde{\mu}_{k}=\mu T Z^{2} \kappa^{-2}, \quad \widetilde{\Delta}_{\lambda}=\Delta_{\lambda} Z^{2} \kappa^{-2}, \quad \widetilde{\Delta}_{\mu}=\Delta_{\mu} Z^{2} \kappa^{-2}, \quad \widetilde{\Delta}_{\kappa}=$ $\Delta_{\kappa} T^{-1} Z \kappa^{-1}$, and $\widetilde{\widetilde{\lambda}}^{\alpha \beta}=Z \widetilde{\sim}^{\alpha \beta}-\widetilde{\Delta}_{\kappa} J^{\alpha \beta}, \widetilde{\mu}^{\alpha \beta}=\tilde{\mu} \delta^{\alpha \beta}-$ $\widetilde{\Delta}_{\mu} J^{\alpha \beta}$, and $\tilde{\lambda}^{\alpha \beta}=\widetilde{\lambda} \delta^{\alpha \beta}-\widetilde{\Delta}_{\lambda} J^{\alpha \beta}$, where $J^{\alpha \beta} \equiv 1 \forall \alpha, \beta$. Note that $\tilde{\mu}$ and $\tilde{\lambda}$ can be used as a measure of the temperature $T$, while $\widetilde{\Delta}_{\kappa}$ diverges at vanishing temperatures. Finally, as usual, one defines the correlation functions $G_{h_{i} h_{j}}(\boldsymbol{q})=$ $\left[\left\langle h_{i}(\boldsymbol{q}) h_{j}(-\boldsymbol{q})\right\rangle\right]$ and $G_{u_{i} u_{j}}(\boldsymbol{q})=\left[\left\langle u_{i}(\boldsymbol{q}) u_{j}(-\boldsymbol{q})\right\rangle\right]$ as well the thermal $\chi(\boldsymbol{q})$ and disorder $C(\boldsymbol{q})$ ones through [24,25]

$$
\begin{aligned}
G_{h_{i} h_{j}}(\boldsymbol{q}) & =\left[\left\langle\delta h_{i}(\boldsymbol{q}) \delta h_{j}(-\boldsymbol{q})\right\rangle\right]+\left[\left\langle h_{i}(\boldsymbol{q})\right\rangle\left\langle h_{j}(-\boldsymbol{q})\right\rangle\right] \\
& \equiv T \chi_{h_{i} h_{j}}(\boldsymbol{q})+C_{h_{i} h_{j}}(\boldsymbol{q})
\end{aligned}
$$

\footnotetext{
${ }^{2}$ This includes the major difference that $c$ couples with the derivative of the order parameter $\Delta \boldsymbol{h}$ and not directly to the order parameter $\partial_{i} \boldsymbol{h}$.
} 
and

$$
\begin{aligned}
G_{u_{i} u_{j}}(\boldsymbol{q}) & =\left[\left\langle\delta u_{i}(\boldsymbol{q}) \delta u_{j}(-\boldsymbol{q})\right\rangle\right]+\left[\left\langle u_{i}(\boldsymbol{q})\right\rangle\left\langle u_{j}(-\boldsymbol{q})\right\rangle\right] \\
& \equiv T \chi_{u_{i} u_{j}}(\boldsymbol{q})+C_{u_{i} u_{j}}(\boldsymbol{q}),
\end{aligned}
$$

with $\delta h_{i}(\boldsymbol{q})=h_{i}(\boldsymbol{q})-\left\langle h_{i}(\boldsymbol{q})\right\rangle, \delta u_{i}(\boldsymbol{q})=u_{i}(\boldsymbol{q})-\left\langle u_{i}(\boldsymbol{q})\right\rangle$. At low momenta, one expects the scaling behaviors $[24,25]$

$$
\begin{array}{ll}
\chi_{h_{i} h_{j}}(\boldsymbol{q}) \sim q^{-(4-\eta)}, & C_{h_{i} h_{j}}(\boldsymbol{q}) \sim q^{-\left(4-\eta^{\prime}\right)}, \\
\chi_{u_{i} u_{j}}(\boldsymbol{q}) \sim q^{-\left(4-\eta_{u}\right)}, & C_{u_{i} u_{j}}(\boldsymbol{q}) \sim q^{-\left(4-\eta_{u}^{\prime}\right)} .
\end{array}
$$

Ward identities relate these quantities [24,25] through $\eta_{u}+$ $2 \eta=4-D$ and $\eta_{u}^{\prime}+2 \eta^{\prime}=4-D$. Finally, one defines [24,25,34], from $\eta$ and $\eta^{\prime}$, the exponent $\phi=\eta^{\prime}-\eta$ that determines which kind of fluctuations - thermal or disorderdominates at a given fixed point: (i) if $\phi>0$, the fixed point behavior is dominated by thermal fluctuations; (ii) if $\phi<0$, the fixed point behavior is dominated by disorder fluctuations; (iii) if $\phi=0$, both fluctuations coexist; the fixed point is said to be marginal.

Renormalization group equations and fixed points. As in the disorder-free $[44,46,47]$ case, Ward identities associated with a partial rotation invariance ensure the renormalizability of the theory. Also, only the renormalizations of phonon and flexural mode propagators are required. As in [44], we have treated the massless theory using the modified minimal subtraction scheme and used standard techniques for computing massless Feynman diagram calculations; see, e.g., [48]. As usual, one defines dimensionless coupling constants $\bar{\mu}=Z^{-2} k^{D-4} \tilde{\mu}$, $\bar{\lambda}=Z^{-2} k^{D-4} \tilde{\lambda}, \bar{\Delta}_{\mu}=Z^{-2} k^{D-4} \widetilde{\Delta}_{\mu}, \bar{\Delta}_{\lambda}=Z^{-2} k^{D-4} \widetilde{\Delta}_{\lambda}$, and $\bar{\Delta}_{\kappa}=Z^{-1} \widetilde{\Delta}_{\kappa}$. The running anomalous dimension is given by $\eta_{t}=\partial_{t} \ln Z$ and $\phi_{t}=\eta_{t}^{\prime}-\eta_{t}=\partial_{t} \ln \bar{\Delta}_{\kappa},{ }^{3}$ where $t=\ln \bar{k}$, with $\bar{k}$ being a renormalization momentum scale. ${ }^{4}$ The RG equations are given in the Supplemental Material [51] and computational details will be given in a forthcoming publication [49]. Note, finally, that our computations have been checked using the effective-field theory obtained by integrating over the Gaussian phonon field $\mathbf{u}[23,34,49,50]$; see below.

Let us first recall the one-loop results [24,25]. At this order, one finds, in $D<4$, two nontrivial physical fixed points located on the hypersurfaces $\bar{\lambda} / \bar{\mu}=\bar{\Delta}_{\lambda} / \bar{\Delta}_{\mu}=-1 / 3$. First is the disorder-free fixed point $P_{4}$, for which $\bar{\mu}=96 \pi^{2} \epsilon /(24+$ $\left.d_{c}\right), \bar{\Delta}_{\mu}=\bar{\Delta}_{\kappa}=0$, and $\eta=\eta^{\prime} / 2=\phi=12 \epsilon /\left(24+d_{c}\right)$. It is fully attractive and thus controls the long-distance behavior of both disordered and disorder-free membranes. This fixed point is, obviously, dominated by thermal fluctuations. There is another fixed point $P_{5}$ located at vanishing temperature, i.e., $\bar{\mu}=0$. To get this fixed point from the RG equations, one has to consider the coupling constant $\bar{g}_{\mu}=\bar{\mu} \bar{\Delta}_{\kappa}$ that stays finite at vanishing temperature while $\bar{\Delta}_{\kappa}$ is diverging. $P_{5}$ is characterized by $\bar{\Delta}_{\mu}=24 \pi^{2} \epsilon / d_{c 6}, \bar{g}_{\mu}=48 \pi^{2} \epsilon / d_{c 6}$, and $\eta=\eta^{\prime}=3 \epsilon / d_{c 6}$ with $d_{c 6}=d_{c}+6$. At this fixed point, one has $\phi=0$; it is thus marginal. A further analysis taking account of nonlinear contributions shows that $P_{5}$ is marginally relevant $[24,25]$.

\footnotetext{
${ }^{3}$ We indicate a misprint in [41] where this relation was incorrectly written $\eta_{t}^{\prime}=\eta_{t}+\partial_{t} \ln \widetilde{\Delta}_{\kappa}$.

${ }^{4} \bar{k}$ is related to $k$ by $\bar{k}^{2}=4 \pi e^{-\gamma_{E}} k^{2}$, where $\gamma_{E}$ is the Euler constant.
}

At two-loop order, we recover the disorder-free fixed point $P_{4}$ whose coordinates and anomalous dimension have been given in [44]. Using the variables relevant to study the vanishing temperature, we also identify a fixed point with $\bar{\mu}=0$ that coincides with the fixed point $P_{5}$ found at one-loop order. Note, however, that we are not able to fully characterize this fixed point; see below. Finally, the search for a new fixed point is inspired by the NPRG results [41], where we recall that the coordinates of $P_{c}$ in the vicinity of $D_{u c}=4$ are given at leading nontrivial order in $\epsilon$ by $[41,42]$ $\bar{\mu}=4 \pi^{2} \epsilon^{2}\left(5 d_{c}+27\right) / 15 d_{c 6}^{2}+O\left(\epsilon^{3}\right), \bar{\lambda}=-1 / 3 \bar{\mu}+O\left(\epsilon^{3}\right)$, $\bar{\Delta}_{\mu}=24 \pi^{2} \epsilon / d_{c 6}+O\left(\epsilon^{2}\right), \quad \bar{\Delta}_{\lambda}=-1 / 3 \bar{\Delta}_{\mu}+O\left(\epsilon^{2}\right), \quad$ and $\bar{\Delta}_{\kappa}=180 d_{c 6} /\left(27+5 d_{c}\right) \epsilon$, while the anomalous dimension is given by

$$
\eta_{c}^{N P R G}=\frac{3 \epsilon}{d_{c 6}}-\frac{d_{c}\left(425 d_{c}+2556\right)}{240 d_{c 6}^{3}} \epsilon^{2},
$$

with $\eta_{c}^{\prime N P R G}=\eta_{c}^{N P R G}$. Within the perturbative context, we thus consider, for the various coupling constants, the ansatz

$$
\bar{X}=\mathcal{C}_{X}^{(1)} \epsilon+\mathcal{C}_{X}^{(2)} \epsilon^{2} \text { for } \bar{X}=\left\{\bar{\lambda}, \bar{\mu}, \bar{\Delta}_{\lambda}, \bar{\Delta}_{\mu}\right\},
$$

where the $\mathcal{C}_{X}^{(1)}$ are given by the coordinates of the vanishing temperature fixed point $P_{5}$ at one-loop order, ${ }^{5}$ and the unusual—singular-behavior for $\bar{\Delta}_{\kappa}$ :

$$
\bar{\Delta}_{\kappa}=C_{\Delta_{\kappa}}^{(-1)} / \epsilon+C_{\Delta_{\kappa}}^{(0)} .
$$

Canceling the RG equations at (next-to-leading) order $\epsilon^{3}$ for the $\bar{X}$ 's and at order $\epsilon$ for $\bar{\Delta}_{\kappa}$, we find a new fixed point $P^{*}$ with parameters

$$
\begin{aligned}
\mathcal{C}_{\lambda}^{(2)} & =-\frac{\mathcal{C}_{\mu}^{(2)}}{3}, \\
\mathcal{C}_{\Delta_{\lambda}}^{(2)} & =\frac{\mathcal{C}_{\mu}^{(2)} d_{c}}{6 d_{c 6}}-\frac{2\left(6 d_{c}+83\right) \pi^{2}}{5 d_{c 6}^{3}}, \\
\mathcal{C}_{\Delta_{\mu}}^{(2)} & =-\frac{\mathcal{C}_{\mu}^{(2)} d_{c}}{2 d_{c 6}}-\frac{6\left(14 d_{c}+37\right) \pi^{2}}{5 d_{c 6}^{3}}, \\
\mathcal{C}_{\Delta_{\kappa}}^{(0)} & =-\frac{2\left(d_{c}+3\right)}{d_{c 6}}-\frac{4\left(28 d_{c}+27\right) \pi^{2}}{5 \mathcal{C}_{\mu}^{(2)} d_{c 6}^{3}}, \\
\mathcal{C}_{\Delta_{\kappa}}^{(-1)} & =\frac{48 \pi^{2}}{\mathcal{C}_{\mu}^{(2)} d_{c 6}} .
\end{aligned}
$$

As seen in these expressions, one of the parameters entering in (9)-(11), here $\mathcal{C}_{\mu}^{(2)}$, is left undetermined. An analysis of the NPRG approach [41] shows that using the ansatz (9) and (10) and canceling the corresponding NPRG equations at the same order in $\epsilon$ leads to the same difficulty, i.e., the same indetermination of $\mathcal{C}_{\mu}^{(2)}$, which is thus a feature of the $\epsilon$ expansion and not of the loop expansion. It is thus judicious to go beyond the former expansion. One can first analyze the RG equations numerically. Doing this, we clearly identify a once-unstable fixed point in the vicinity of $D=4$ with coordinates of the type (9)-(11). Thereafter, in order to analytically

\footnotetext{
${ }^{5}$ The coordinates of $P_{5}$ only differ from those of $P_{c}$ by terms of order $\epsilon^{2}$.
} 
identify this fixed point, one can push the solution of the RG equations beyond next-to-leading order, notably by canceling the equation for $\bar{\Delta}_{\kappa}$ at order $\epsilon^{2}$. This raises the indetermination on $\mathcal{C}_{\mu}^{(2)}$, which is found to be equal to

$$
C_{\mu, 2 f}^{(2)}=\frac{4 \pi^{2}\left(3075 d_{c}^{2}+16850 d_{c}-576\right)}{15 d_{c 6}^{2}\left(166+169 d_{c}+20 d_{c}^{2}\right)},
$$

where the index $2 f$ refers to the two-field (phonon-flexuron) theory. Note that this value is approximate as one expects a three-loop contribution to (12). However, with the expressions (11) and (12), we very satisfactorily reproduce the numerical results in the extreme vicinity of $D=4$, e.g., for $\epsilon$ of order $10^{-3}$, the errors for the coordinates are, at worst, of order $10^{-8}$. We now give the eigenvalues around $P^{*}$ at leading nonvanishing order,

$$
\left\{\frac{3 d_{c} \mathcal{C}_{\mu}^{(2)}}{8 d_{c 6}^{3}} \epsilon^{2} ;-\frac{d_{c}}{d_{c 6}} \epsilon ;-\frac{d_{c}}{d_{c 6}} \epsilon ;-\epsilon ;-\epsilon\right\},
$$

with $\mathcal{C}_{\mu}^{(2)}$ given by (12), which is positive for any physical value of $d_{c}$. Having one repulsive direction, the fixed point $P^{*}$ is associated with a second-order phase transition. It is characterized by the anomalous dimensions,

$\eta_{c}^{2 l}=\frac{3 \epsilon}{d_{c 6}}-\frac{d_{c}\left[5 \mathcal{C}_{\mu}^{(2)} d_{c 6}^{2}+2\left(407+60 d_{c}\right) \pi^{2}\right]}{80 \pi^{2} d_{c 6}^{3}} \epsilon^{2}$,

and $\eta_{c}^{\prime}=\eta_{c}$ implying $\phi=0$ so that $P^{*}$ is marginal. The result (13) is also found within the effective (pure flexuron) approach of the theory (see the Supplemental Material [51] and [49]), which is a strong confirmation of the validity of our result. Note, however, that in the latter case, the approximate expression of $\mathcal{C}_{\mu}^{(2)}$ slightly differs and is given by

$$
C_{\mu, \mathrm{eff}}^{(2)}=\frac{4 \pi^{2}\left(3450 d_{c}^{2}+19100 d_{c}-576\right)}{15 d_{c 6}^{2}\left(166+169 d_{c}+20 d_{c}^{2}\right)} .
$$

However, this change affects the physical results extremely weakly; see below.

All the qualitative properties of $P^{*}$-one marginally relevant direction of order $\epsilon^{2}$, and one coupling constant $\mu$ of order $\epsilon^{2}$-are shared with those of the fixed point $P_{c}$ found in $[41,42]$ using a NPRG approach. Moreover, the agreement between the anomalous dimension obtained within the present work, i.e., (13) with $\mathcal{C}_{\mu}^{(2)}$ given by (12) or (14), and that computed with the NPRG approach (8) is remarkable; see Fig. 1 where we have represented the two-loop corrections $\eta_{c}^{(2)}$ defined as $\eta_{c}=\eta_{c}^{(1)}+\eta_{c}^{(2)} \epsilon^{2}$ as functions of $d_{c}$. In the physical situation $d_{c}=1$, they are given by $\eta_{c}^{(2) N P R G}=0.0362$, $\eta_{c, 2 f}^{(2) 2 l}=0.0366$, and $\eta_{c, \text { eff }}^{(2) 21}=0.0370$. We thus identify $P^{*}$ with $P_{c}$ and fully confirm the existence of a-wrinkling-phase transition at finite temperature.

Concerning the fixed point $P_{5}$, we find, numerically, that it is, in fact, marginally irrelevant-in agreement with the unstable character of $P_{c}$ and with the NPRG approach. However, as said above, there are difficulties to characterize $P_{5}$, as well as $P_{c}$, at low temperatures. Indeed, this implies the use of the low-temperature variables $\bar{g}_{\lambda}=\bar{\lambda} \bar{\Delta}_{\kappa}$ and $\bar{g}_{\mu}=\bar{\mu} \bar{\Delta}_{\kappa}$ at order $\epsilon^{2}$. But, in the vicinity of $P_{5}$ or $P_{c}$, one has, at next-to-leading

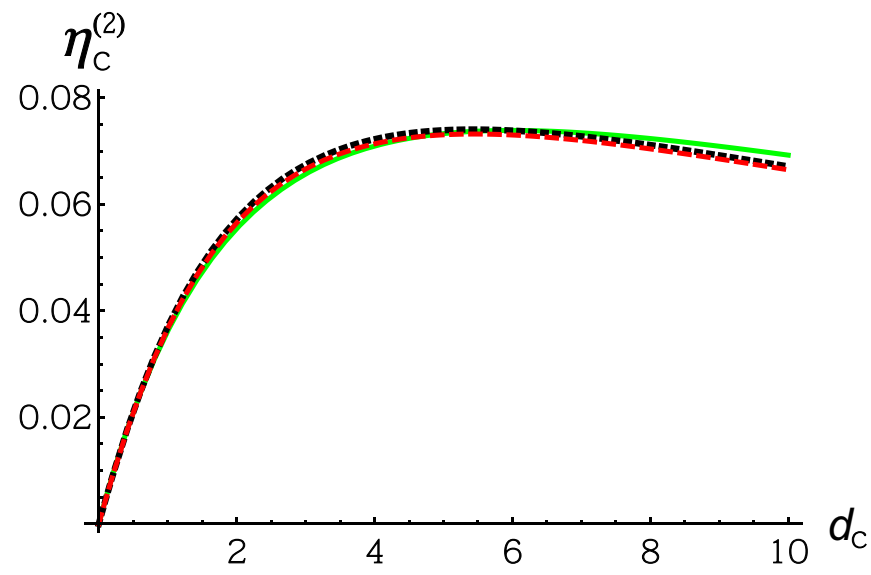

FIG. 1. The correction of $O\left(\epsilon^{2}\right)$ to $\eta_{c}, \eta_{c}^{(2)}$, as a function of $d_{c}$ at the fixed point $P_{c}$. The solid line shows the prediction from the NPRG approach [41]; the dashed line shows the two-loop, two-field result (present work); the dotted line shows the two-loop, effective result (present work).

order in $\epsilon$,

$$
\bar{g}_{\mu}=\mathcal{C}_{\mu}^{(2)} \mathcal{C}_{\Delta_{\kappa}}^{(-1)} \epsilon+\mathcal{C}_{\mu}^{(3)} \mathcal{C}_{\Delta_{\kappa}}^{(-1)} \epsilon^{2}+\mathcal{C}_{\mu}^{(2)} \mathcal{C}_{\Delta_{\kappa}}^{(0)} \epsilon^{2}+O\left(\epsilon^{3}\right),
$$

where it appears that due to the specific scaling of $\bar{\Delta}_{\kappa}$ with $\epsilon$ that involves negative powers of this parameter, the subsubleading contribution in $\epsilon$ to $\mu$, i.e., $\mathcal{C}_{\mu}^{(3)}$, is needed but is obviously lacking within the present, two-loop order computation.

Conclusion. We have investigated quenched disordered membranes by means of a two-loop order perturbative approach. As a main result, our approach clearly confirms the finding obtained with the NPRG approach [41], i.e., the existence of a richer phase diagram than that expected from previous investigations: the existence of a novel fixed point $P_{c}$ characterizing a wrinkling phase transition occurring at a temperature $T_{c}$ separating a disorder-free phase at $T>T_{c}$ controlled by the vanishing-disorder attractive fixed point $P_{4}$ and a low-temperature $T<T_{c}$ "glassy phase" controlled by the vanishing-temperature, finite-disorder, attractive fixed point $P_{5}$. We thus have reached a consistent picture of disordered membranes at finite temperatures and, in particular, of the occurrence of a wrinkling transition. Our work reinforces the interest to investigate experimentally or numerically this transition in several systems involving both curvature and stretching disorder. This includes (i) a further study of partially polymerized fluid vesicles that have already been investigated by Chaieb et al. and have shown to be qualitatively and quantitatively well explained by the scenario proposed in $[41,42]$, and (ii) a careful investigation of graphene and graphenelike materials with quenched lattice defects. Moreover, our work, by confirming the attractive character of the vanishing-temperature fixed point $P_{5}$, opens the possibility of a low-temperature phase controlled by a complex energy landscape and a genuine glassy phase that have been intensively looked for theoretically. It is thus pressing to probe this phase experimentally and numerically, notably in the context of the physics of graphene where it would be of prime interest to study the effects induced by disorder on the electronic and transport properties of graphene and graphenelike materials 
in this phase. Finally, from a more formal point of view, our work strongly suggests to deeply investigate the nature of the perturbative series in the vicinity of the fixed points $P_{5}$ and $P_{c}$. In particular it would be of interest, even if it would represent a very substantial amount of work, to see whether the three-loop contributions indeed raise the ambiguities encountered within the two-loop order computation when studying the wrinkling transition.

Acknowledgments. We wish to greatly thank S. Teber for discussions.
[1] L. Radzihovsky, in Proceedings of the Fifth Jerusalem Winter School for Theoretical Physics, 2nd ed. (World Scientific, Singapore, 2004).

[2] V. S. Dotsenko, Introduction to the Replica Theory of Disordered Statistical Systems (Cambridge University Press, Cambridge, 2001).

[3] Spin Glasses and Random Fields, edited by A. P. Young (World Scientific, Singapore, 1998).

[4] T. Nattermann, Spin Glasses and Random Fields (World Scientific, Singapore, 1999).

[5] C. De Dominicis, I. Kondor, and T. Temesvari, in Spin Glasses and Random Fields, edited by A. P. Young (World Scientific, Singapore, 1998).

[6] C. De Dominicis and I. Giardina, Random Fields And Spin Glasses: A Field Theory Approach (Cambridge University Press, Cambridge, 2006).

[7] K. W. Wiese and P. L. Doussal, Markov Proc. Relat. Fields 13, 777 (2007).

[8] G. Tarjus and M. Tissier, Eur. Phys. J. B 93, 50 (2020).

[9] M. Mutz, D. Bensimon, and M. J. Brienne, Phys. Rev. Lett. 67, 923 (1991).

[10] S. Chaieb, V. K. Natrajan, and A. A. El-rahman, Phys. Rev. Lett. 96, 078101 (2006).

[11] S. Chaieb, S. Málková, and J. Lal, J. Theor. Biol. 251, 60 (2008).

[12] L. Radzihovsky and D. R. Nelson, Phys. Rev. A 44, 3525 (1991).

[13] F. Banhart, J. Kotakoski, and A. Krasheninnikov, ACS Nano 5, 26 (2011).

[14] L. Liu, M. Qing, Y. Wang, and S. Chen, J. Mater. Sci. Technol. 31, 599 (2015).

[15] G. Yang, L. Li, W. B. Lee, and M. C. Ng, Sci. Technol. Adv. Mater. 19, 613 (2018).

[16] In Handbook of Graphene: Physics, Chemistry, and Biology, Vol. 2, edited by T. Stauber (John Wiley \& Sons, 2019), p. I.

[17] K. S. Novoselov, A. K. Geim, S. V. Morozov, D. Jiang, Y. Zhang, S. V. Dubonos, I. V. Gregorieva, and A. A. Firsov, Science 306, 666 (2004).

[18] K. S. Novoselov, A. K. Geim, S. V. Morozov, D. Jiang, M. I. Katsnelson, I. V. Gregorieva, S. V. Dubonos, and A. A. Firsov, Nature (London) 438, 197 (2005).

[19] J. Kotakoski, A. V. Krasheninnikov, U. Kaiser, and J. C. Meyer, Phys. Rev. Lett. 106, 105505 (2011).

[20] F. R. Eder, J. Kotakoski, U. Kaiser, and J. C. Meyer, Sci. Rep. 4, 4060 (2014).

[21] W.-J. Joo et al., Sci. Adv. 3, e1601821 (2017).

[22] D. R. Nelson and L. Radzihovsky, Europhys. Lett. 16, 79 (1991).

[23] L. Radzihovsky and P. Le Doussal, J. Phys. I (France) 2, 599 (1992).
[24] D. C. Morse, T. C. Lubensky, and G. S. Grest, Phys. Rev. A 45, R2151 (1992).

[25] D. C. Morse and T. C. Lubensky, Phys. Rev. A 46, 1751 (1992).

[26] D. Bensimon, D. Mukamel, and L. Peliti, Europhys. Lett. 18, 269 (1992).

[27] D. Bensimon, M. Mutz, and T. Gulik, Physica A 194, 190 (1993).

[28] R. Attal, S. Chaieb, and D. Bensimon, Phys. Rev. E 48, 2232 (1993).

[29] Y. Park and C. Kwon, Phys. Rev. E 54, 3032 (1996).

[30] S. Mori, Phys. Rev. E 54, 338 (1996).

[31] A. Benyoussef, D. Dohmi, A. E. Kenz, and L. Peliti, Eur. Phys. J. B 6, 503 (1998).

[32] P. Le Doussal and L. Radzihovsky, Phys. Rev. B 48, 3548 (1993).

[33] S. Mori and M. Wadati, Phys. Lett. A 185, 206 (1994).

[34] P. Le Doussal and L. Radzihovsky, Ann. Phys. (NY) 392, 340 (2018).

[35] J.-P. Kownacki and D. Mouhanna, Phys. Rev. E 79, 040101(R) (2009).

[36] F. L. Braghin and N. Hasselmann, Phys. Rev. B 82, 035407 (2010).

[37] K. Essafi, J.-P. Kownacki, and D. Mouhanna, Phys. Rev. Lett. 106, 128102 (2011).

[38] N. Hasselmann and F. L. Braghin, Phys. Rev. E 83, 031137 (2011).

[39] K. Essafi, J.-P. Kownacki, and D. Mouhanna, Phys. Rev. E 89, 042101 (2014)

[40] O. Coquand and D. Mouhanna, Phys. Rev. E 94, 032125 (2016).

[41] O. Coquand, K. Essafi, J.-P. Kownacki, and D. Mouhanna, Phys. Rev. E 97, 030102(R) (2018).

[42] O. Coquand, K. Essafi, J.-P. Kownacki, and D. Mouhanna, Phys. Rev. E 101, 042602 (2020).

[43] D. R. Saykin, V. Yu. Kachorovskii, and I. S. Burmistrov, Phys. Rev. Research 2, 043099 (2020).

[44] O. Coquand, D. Mouhanna, and S. Teber, Phys. Rev. E 101, 062104 (2020)

[45] A. Mauri and M. I. Katsnelson, Nucl. Phys. B 956, 115040 (2020).

[46] E. Guitter, F. David, S. Leibler, and L. Peliti, Phys. Rev. Lett. 61, 2949 (1988).

[47] E. Guitter, F. David, S. Leibler, and L. Peliti, J. Phys. (Paris) 50, 1787 (1989).

[48] A. V. Kotikov and S. Teber, Phys. Part. Nucl. 50, 1 (2019).

[49] O. Coquand and D. Mouhanna (unpublished).

[50] D. R. Nelson and L. Peliti, J. Phys. (Paris) 48, 1085 (1987).

[51] See Supplemental Material at http://link.aps.org/supplemental/ 10.1103/PhysRevE.103.L031001 for the two-loop renormalization group equations for both the two-field (phononflexuron) and the effective (pure flexuron) theory of disordered polymerized membranes. 